\title{
Resistencia enzimática en Pseudomonas aeruginosa, aspectos clínicos y de laboratorio
}

\section{Enzymatic resistance in Pseudomonas aeruginosa, clinical and laboratory aspects}

\author{
Diana Isabel Espinoza Pesantez y German Francisco Esparza Sanchez²
}

'Universidad de Cuenca, Ecuador.
${ }^{2}$ Programa de aseguramiento de la calidad en el laboratorio clínico PROASECAL, Colombia.

Declaración de conflictos de interés: Ninguno

Financiamiento: Ninguno

Recibido: 4 de enero de 2020 (segunda versión: 2-de octubre de 2020) / Aceptado: 9 de diciembre de 2020

\section{Resumen}

Pseudomonas aeruginosa es uno de los principales patógenos que causa infecciones asociadas a la atención en salud (IAAS). Su capacidad de adaptación, diseminación, resistencia intrínseca a los antimicrobianos y de adquirir nuevos mecanismos a través de elementos genéticos móviles, hacen que el tratamiento de las infecciones por este microorganismo sea un desafío para el médico clínico. Intrínsecamente, $P$. aeruginosa, presenta una reducida permeabilidad en la membrana externa, debido a la expresión de bombas de expulsión, y una cefalosporinasa tipo AmpC inducible. Además, $P$. aeruginosa es capaz de adquirir nuevos determinantes de resistencia por transferencia horizontal en forma de casetes situados en integrones, y a su vez, localizados en transposones o plásmidos. Dentro de la resistencia enzimática que presenta $P$. aeruginosa destacan las $\beta$-lactamasas, incluyendo aquellas de espectro extendido (BLEE) y las carbapenemasas. Pero también enzimas modificadoras de los aminoglucósidos, haciendo que este microorganismo pueda presentar fenotipos de multi-resistencia (MDR), resistencia extrema (XDR) y panresistencia (PDR) a los antimicrobianos denominados antipseudomonas, incluyendo a las nuevas cefalosporinas con inhibidores de $\beta$-lactamasas.

Palabras clave: Pseudomonas aeruginosa; resistencia antimicrobiana, resistencia enzimática; nuevos antimicrobianos; $\beta$-lactámicos; aminoglucósidos; inhibidores de $\beta$-lactamasas.

\section{Abstract}

Pseudomonas aeruginosa is one of the major pathogens causing healthcare-associated infections (HAI). Its capacity of adaptation, dissemination, intrinsic resistance to antimicrobials and of acquiring new mechanisms through mobile genetic elements, make the treatment of infections by this microorganism a challenge for the clinician. Intrinsically, $P$. aeruginosa, presents a reduced permeability in the external membrane, due to the expression of efflux pumps, and an inducible AmpC-type cephalosporinase. In addition, $P$. aeruginosa is able to acquire new resistance determinants by horizontal transfer in the form of cassettes located in integrons, and in turn located in transposons or plasmids. Within the enzymatic resistance that $P$. aeruginosa presents, betalactamases, including extended spectrum (ESBL) and carbapenemases. But also aminoglycoside modifying enzymes, stand out, causing this microorganism to present multi-resistance phenotypes (MDR), extreme resistance (XDR) and pan-resistance (PDR) to the called antipseudomonal antibiotics, including the new cephalosporins with betalactamase inhibitors.

Keywords: Pseudomonas aeruginosa; antibiotic resistance; enzymatic resistance; new antimicrobials; betalactams; aminoglycosides; betalactamase inhibitors.

\footnotetext{
Correspondencia a:

Diana Isabel Espinoza Pesantez

diespinoza@javeriana.edu.co
} 


\section{Introducción}

$P$ seudomonas aeruginosa, es un bacilo gramnegativo, no fermentador de glucosa y lactosa, no forma parte de la microbiota normal del ser humano, pero puede encontrarse como colonizante de las zonas corporales húmedas (axilas, conducto auditivo, región perianal y mucosas) ${ }^{1}$. Es uno de los principales patógenos que causan infecciones asociadas a la atención en salud (IAAS), afectando particularmente a pacientes inmunocomprometidos (pacientes con dispositivos invasivos, post-quirúrgicos, hemato-oncológicos, neutropénicos, con quemaduras graves, etc. $)^{2,3}$.

La creciente prevalencia de IAAS por cepas de $P$. aeruginosa multi-resistente (MDR), debido a su resistencia intrínseca y la capacidad de adquirir mecanismos adicionales de resistencia a una gran variedad de antimicrobianos inclusive durante la terapia, limita las opciones terapéuticas disponibles, lo que se asocia a una elevada morbi-mortalidad, en especial en pacientes que reciben una terapia empírica inadecuada $(30,7 \%)$, comparativamente con aquellos que reciben un manejo apropiado $(17,8 \%)^{2,4}$. Así mismo, la elevada prevalencia de $P$. aeruginosa con resistencia a carbapenémicos, fluoroquinolonas y aminoglucósidos presenta desafíos significativos para los profesionales de salud. La ototoxicidad y la toxicidad renal, además de la creciente tasa de resistencia de $P$. aeruginosa a aminoglucósidos, limita el potencial terapéutico de las combinaciones con $\beta$-lactámicos para infecciones por $P$. aeruginosa $\mathrm{MDR}^{2,5}$.

\section{Patogenia}

La patogénesis de la infección causada por $P$. aeruginosa ocurre en tres etapas: La primera es la adhesión bacteriana y colonización, la segunda etapa es invasión local, y por último la diseminación e infección sistémica. Tras el establecimiento de la infección, $P$. aeruginosa secreta exotoxinas del tipo A y S conjuntamente con enzimas hidrolíticas, compuestos que al entrar en contacto con los tejidos degradan las membranas celulares y las destruyen progresivamente con el objetivo de facilitar su diseminación, la invasión tisular y la necrosis ${ }^{6,7}$

La gravedad de las infecciones causadas por $P$. aeruginosa se debe a los factores de virulencia que presenta, principalmente exotoxinas, proteasas y exo-enzimas, que causan daño extenso del tejido del hospedero al alterar la estructura normal del cito-esqueleto, ocasionando una despolimerización de los filamentos de actina y la escisión de las inmunoglobulinas $\mathrm{G}$ y $\mathrm{A}^{8,9}$.

\section{Resistencia a antimicrobianos en $P$. aeruginosa}

\section{Mecanismos de resistencia intrínseca}

La resistencia intrínseca es definida como la resistencia antimicrobiana innata o natural que se observa en la mayoría de aislados de una especie. Pseudomonas aeruginosa expresa un alto nivel de resistencia intrínseca a la mayoría de los antimicrobianos, debido principalmente, a una membrana externa poco permeable, expresión de bombas de expulsión, mutaciones que modifican el sitio de acción de algunos antimicrobianos y a la inactivación enzimática a través de una cefalosporinasa cromosomal tipo AmpC inducible ${ }^{1,3}$.

De acuerdo con la guía CLSI M100 edición 30th, la resistencia natural a los antimicrobianos en este microorganismo se describe en la Tabla $1^{10}$.

\section{Mecanismos de resistencia adquirida}

Pseudomonas aeruginosa puede desarrollar resistencia a los antimicrobianos a través de mutaciones en los genes

\section{Tabla 1. Resistencia antimicrobiana intrínseca en $P$. aeruginosa}

\begin{tabular}{|c|c|c|c|c|}
\hline \multirow[t]{5}{*}{$\beta$-lactámicos } & Penicilinas y combinaciones de $\beta$-lactámicos & \multicolumn{3}{|c|}{ Ampicilina, amoxicilina, ampicilina/sulbactam, amoxicilina/ácido clavulánico } \\
\hline & Cefalosporinas de $1^{\circ}$ generación & \multicolumn{3}{|c|}{ Cefazolina, cefalotina, cefradina, cefadroxilo, cefalexina } \\
\hline & Cefalosporinas de $2^{\circ}$ generación y cefamicinas & \multicolumn{3}{|c|}{ Cefuroxima, cefoxitina, cefotetan } \\
\hline & Cefalosporinas de $3^{\circ}$ generación & \multicolumn{3}{|c|}{ Ceftriaxona, cefotaxima } \\
\hline & Carbapenémicos & \multicolumn{3}{|l|}{ Ertapenem } \\
\hline $\begin{array}{l}\text { Tetraciclinas y glicilci- } \\
\text { clinas }\end{array}$ & Quinolonas & Anfenicoles & Nitrofuranos & $\begin{array}{c}\text { Sulfonamidas asociadas a } \\
\text { trimetoprima }\end{array}$ \\
\hline $\begin{array}{l}\text { Tetraciclina, doxiciclina, } \\
\text { minociclina, tigeciclina }\end{array}$ & Ácido nalidíxico, ácido pipemídico & Cloranfenicol & Nitrofurantoína & $\begin{array}{c}\text { Trimetoprima/sulfametoxazol } \\
\text { (cotrimoxazol) }\end{array}$ \\
\hline
\end{tabular}

Fuente: guía CLSI M100 30 edición Apéndice B -Tabla B-2 ${ }^{10}$. 
propios o de adquisición horizontal por elementos genéticos móviles que transportan enzimas como $\beta$-lactamasas de espectro extendido (BLEE), carbapenemasas de clase A y D de Ambler y metalo- $\beta$-lactamasas (MBL) de clase $\mathrm{B}$ de Ambler ${ }^{6}$. Adicional a estos mecanismos, $P$. aeruginosa puede adquirir genes que codifican enzimas modificadoras de los aminoglucósidos (EMA); todo esto sumado a mecanismos intrínsecos de resistencia, como bombas de expulsión e impermeabilidad de membrana, potencia la resistencia antimicrobiana reduciendo las alternativas terapéuticas ${ }^{9}$.

\section{Resistencia enzimática a los antimicrobianos $\beta$-lactámicos}

\section{B-lactamasas de P. aeruginosa}

Los análisis moleculares y genéticos de aislados clínicos han demostrado que $P$. aeruginosa puede producir una diversidad de $\beta$-lactamasas, algunas de las cuales están codificadas por genes cromosómicos y otras son transportadas por elementos genéticos móviles (como plásmidos y transposones). La resistencia asociada a $\beta$-lactamasas en $P$. aeruginosa depende también de la eficacia de penetración del fármaco, la capacidad de este patógeno para minimizar la acumulación del fármaco en el espacio periplásmico (mediante bombas de expulsión) y los efectos cooperativos de diferentes $\beta$-lactamasas dentro de la misma célula ${ }^{11}$.

A continuación, se resumen las diferentes familias de $\beta$-lactamasas según la clasificación actualizada de Bush y Jacoby (Tabla 2).

\section{Grupo 1. Cefalosporinasa cromosomal AmpC}

Es una $\beta$-lactamasa tipo serina de clase molecular $\mathrm{C}$, se caracteriza por su hidrólisis eficaz de las cefalosporinas y su resistencia a la inactivación por clavulanato, sulbactam y tazobactam. La AmpC de P. aeruginosa es una enzima inducible. En ausencia de otros mecanismos de resistencia, las cepas salvajes de $P$. aeruginosa con producción basal de AmpC, pueden ser susceptibles a las penicilinas antipseudomonas, combinaciones de

Tabla 2. $\beta$-lactamasas de $P$. aeruginosa, según la clasificación actualizada de Bush y Jacoby

\begin{tabular}{|c|c|c|}
\hline $\begin{array}{l}\text { Grupo funcional } \\
\text { Clase molecular }\end{array}$ & Espectro de hidrólisis & Enzimas representativas \\
\hline $\begin{array}{l}\text { Grupo } 1 \\
\text { C }\end{array}$ & $P E N, P E N+I B L, C E F, C F M, M N B$ & AmpC cromosomal \\
\hline $\begin{array}{l}\text { Grupo 1e } \\
\text { C }\end{array}$ & PEN, PEN+IBL, CEF, CFM, MNB, CRB & AmpC de espectro extendido \\
\hline $\begin{array}{l}\text { Grupo } 2 b \\
\text { A }\end{array}$ & PEN, CF1. & TEM-1, TEM-2. SHV-1 \\
\hline $\begin{array}{l}\text { Grupo } 2 \text { be } \\
\text { A }\end{array}$ & PEN, CF1, CFA, MNB & $\begin{array}{l}\text { TEM-4, TEM-21, TEM-24, TEM-42, TEM-116, SHV-2, SHV-2a, SHV-5, SHV-12. CTX-M1 a } \\
\text { CTX-M3, CTX-M14, CTX-M15, CTX-M-43, PER-1, PER-2, VEB-1 a VEB-3, GES-1, GES-2, } \\
\text { GES-5, GES-8, GES-9, GES-13, BEL-1 a BEL-3, PME-1 }\end{array}$ \\
\hline $\begin{array}{l}\text { Grupo 2c } \\
\text { A }\end{array}$ & PEN, PEN + IBL & PSE-1 a PSE-5, CARB-3, CARB-4 \\
\hline $\begin{array}{l}\text { Grupo } 2 d \\
\text { D }\end{array}$ & PEN, PEN + IBL & OXA-5, OXA-6, OXA-10, OXA-13, OXA-20, OXA-46, OXA-56, LCR-1 \\
\hline $\begin{array}{l}\text { Grupo 2de } \\
\text { D }\end{array}$ & PEN, PEN + IBL, CEF, MNB & $\begin{array}{l}\text { OXA-11, OXA-14 a OXA-19, OXA-28, OXA-31, OXA-32, OXA-34 a OXA-36, OXA-45, } \\
\text { OXA-74, OXA-141, OXA-142, OXA-145, OXA-147, OXA-183 }\end{array}$ \\
\hline $\begin{array}{l}\text { Grupo } 2 \mathrm{df} \\
\text { D }\end{array}$ & PEN, PEN + IBL, CFA, CRB & OXA-40, OXA-48, OXA-50, OXA-181, OXA-198 \\
\hline Grupo $2 f$ & PEN, PEN + IBL, CEF, CRB & KPC-2, KPC-5, GES-2, GES-5, GES-18. \\
\hline $\begin{array}{l}\text { Grupo } 3 \\
\text { B }\end{array}$ & PEN, PEN + IBL, CEF, CFM, CRB & $\begin{array}{l}\text { IMP-1, IMP-2, IMP-4 a IMP-7, IMP-9 a IMP-11, IMP-13 a IMP-16, IMP-19 a IMP-26, IMP- } \\
\text { 48, VIM-1 a VIM-11, VIM-13 a VIM-20, VIM-28 a VIM-38, NDM-1, SPM-1, GIM-1, FIM-1 }\end{array}$ \\
\hline \multicolumn{3}{|c|}{$\begin{array}{l}\text { Fuente: Wolter D, y cols. } 2012^{11} \text {. Potron A, y cols. } 2015^{12} \text {. Subedi D, y cols. } 2018^{13} \text {. Shaikh S, y cols. } 2015^{14} \text {. Bonnin R, y cols. } 2018^{15} \text {. Abreviaciones: PEN: penicilinas, } \\
\text { PEN+IBL: penicilinas+inhibidores de } \beta \text {-lactamasas, CEF: cefalosporinas, CFM: cefamicinas, MNB: monobactámicos, CRB: carbapenémicos CF1: cefalosporinas de primera } \\
\text { generación, CEFEE: cefalosporinas de espectro expandido, CFA: cefalosporinas de amplio espectro, GES: Guiana extended spectrum, KPC: Klebsiella pneumoniae carba- } \\
\text { penemase, IMP: active on imipenem, NDM: New Delhi metallo-betalactamase, OXA: oxacilinase, VIM: Verona integron-encoded metallo-betalactamase, GIM: German } \\
\text { imipenemase, SHV: sulfhydryl variable, TEM: Tem oneira, PER: Pseudomonas extended resistant, PSE: Pseudomonas-specific enzymes. }\end{array}$} \\
\hline
\end{tabular}


penicilina + inhibidor, cefalosporinas y carbapenémicos. Sin embargo, la sobre-expresión de AmpC puede conducir a una resistencia a prácticamente todos los $\beta$-lactámicos (principalmente resistencia a ceftazidima), exceptuando los carbapenémicos, donde se requiere la cooperación de mecanismos de resistencia adicionales como bombas de expulsión, disminución en la permeabilidad de membrana por mutaciones en las porinas (OprD ) y/o co-producción de carbapenemasas ${ }^{16,17}$.

El camino hacia la desrepresión de $\mathrm{AmpC}$ involucra tres estructuras genéticas: (i) una permeasa de membrana interna conocida como AmpG; (ii) una amidasa citosólica, AmpD; y (iii) un factor de transcripción, AmpR, que pertenece a la familia de proteínas reguladoras LysR. Estas tres proteínas son necesarias para la inducción del gen amp $C$, tanto en Enterobacterales como en $P$. aeruginosa, aunque no hay evidencia directa que vincule a AmpG con la ruta de inducción de AmpC de $P$. aeruginosa, y estos eventos mutacionales pueden conducir a la aparición de resistencia durante la terapia antimicrobiana ${ }^{18}$. Se ha informado la aparición de resistencia debido a la desrepresión de AmpC en hasta $56 \%$ de los pacientes tratados con penicilinas antipseudomonas, combinaciones de penicilina + inhibidor, cefalosporinas de espectro extendido y aztreonam, y es más frecuente durante el tratamiento de infecciones fuera del tracto urinario y en pacientes con fibrosis quística y neutropenia ${ }^{19}$.

\section{Grupo 1e: AmpC de espectro extendido}

La sobreexpresión de AmpC en sí misma, no afecta la susceptibilidad de $P$. aeruginosa a los carbapenémicos; sin embargo, las variantes mutacionales recientemente descritas de AmpC (cefalosporinasa AmpC de espectro extendido) se han caracterizado por influir directamente en la susceptibilidad a estos antimicrobianos. Aunque estas enzimas pueden exhibir una mayor actividad hidrolítica sobre cefalosporinas e imipenem, la sobreproducción de AmpC de espectro extendido parece ser un requisito para la resistencia a carbapenémicos ${ }^{11}$. Estas enzimas son inhibidas por ceftazidima/avibactam, aunque estudios han demostrado que ceftolozano/tazobactam tiene mayor estabilidad frente a estas enzimas ${ }^{20}$.

\section{Grupo $2 b$}

Son $\beta$-lactamasas tipo serina de clase molecular A, Las $\beta$-lactamasas hidrolizan fácilmente las penicilinas $\mathrm{y}$ cefalosporinas de primera generación ${ }^{17}$. Incluyen las enzimas comunes codificadas por plásmidos TEM-1, TEM-2 y SHV-1. La primera $\beta$-lactamasa de tipo TEM, producida por una cepa de Escherichia coli, se informó en 1965 y más tarde fue llamada TEM-1; una variante de esta enzima, TEM-2, originalmente observada en $P$. aeruginosa, difiere de TEM-1 sólo en un aminoácido, pero no en su perfil de sustrato ${ }^{17,22}$.

\section{Grupo 2 be}

Este grupo incluye una amplia variedad de $\beta$-lactamasas tipo serina de espectro extendido (BLEE) de clase molecular A. Las BLEE son un grupo de $\beta$-lactamasas que hidrolizan penicilinas, cefalosporinas de primera generación, oximino-cefalosporinas (ceftazidima, ceftriaxona y cefotaxima), cefepime y aztreonam; y son inhibidas eficientemente por inhibidores de $\beta$-lactamasas, como ácido clavulánico, sulbactam, tazobactam y ceftazidima/avibactam ${ }^{17,20}$. La primera BLEE derivada de TEM notificada en $P$. aeruginosa fue TEM-42 de un aislado clínico en Francia; desde ese primer informe, sólo se han descrito otras cuatro BLEEs derivadas de TEM (TEM-4, TEM-21, TEM-24 y TEM$116)^{16,18}$. Además, se han identificado cuatro BLEEs derivadas de SHV (SHV-2, SHV-2a, SHV-5 y SHV-12) en aislados clínicos de $P$. aeruginos ${ }^{13}$. Mientras que, de la familia CTX-M, hasta la fecha, se han identificado en $P$. aeruginosa, las siguientes variantes CTX-M1, CTX-M2, CTX-M3, CTX-M14, CTX-M15, CTX-M43 $3^{11-13,23,24}$. Las BLEE de tipo PER comparten sólo alrededor de 25-27\% de homología con las BLEE conocidas de tipo TEM y SHV; PER-1 se detectó por primera vez en $P$. aeruginosa en Turquía, se encontró que $11 \%$ de $P$. aeruginosa produce PER-1. Se ha detectado PER-2, que comparte un $86 \%$ de homología con PER-1, en Salmonella entérica serovar Typhimurium, E. coli, Klebsiella pneumoniae, Proteus mirabilis y Vibrio cholerae ${ }^{14}$. La presencia del gen PER-1 también se ha descrito en Italia, donde se detectó en 9 de 26 aislados del torrente sanguíneo de $P$. aeruginosa resistente a ceftazidima ${ }^{25}$.

La detección de BLEEs en $P$. aeruginosa representa un riesgo diagnóstico ante la ausencia de pruebas fenotípicas estandarizadas. Adicionalmente, el impacto clínico de la producción de estas enzimas en $P$. aeruginosa es incierto, por lo que informarlas en el antibiograma mediante pruebas fenotípicas no estandarizadas internacionalmente o editar a resistente el resultado de cefalosporinas o penicilinas no se encuentra recomendado y puede generar un consumo innecesario y exagerado de carbapenémicos en $P$. aeruginosa, lo cual va en contravía de los programas de uso apropiado de antimicrobianos (PROA) ${ }^{26}$.

\section{Grupo $2 c$}

Son penicilinasas tipo serina, de clase molecular A. Este tipo de enzimas identificadas en $P$. aeruginosa incluyen PSE-1, PSE-2, PSE-3, PSE-4, PSE-5, CARB-3 y CARB-4 ${ }^{17}$; aunque estas enzimas son inhibidas por el clavulanato, hidrolizan la ticarcilina con tal eficacia que pueden proporcionar a $P$. aeruginosa resistencia a ticarcilina/clavulanato. En contraste, las cepas de $P$. aeruginosa que producen enzimas tipo PSE son más susceptibles a piperacilina/tazobactam ${ }^{15}$. 
también codifica una enzima cromosómica OXA-50, cuyas variantes alélicas se han descrito y caracterizado como carbapenemasas ${ }^{(12)}$. Carbapenemasa OXA-48 y OXA-181 se aislaron en la India y en Reino Unido (de un paciente repatriado de la India), respectivamente, y una nueva carbapenemasa, OXA-198, es una $\beta$-lactamasa de clase D codificada por plásmidos, involucrada en la resistencia a carbapenémicos en un aislado de $P$. aeruginosa en Bélgica ${ }^{15}$.

\section{Grupo 2f: Carbapenemasas}

Son $\beta$-lactamasas tipo serina que pertenecen a la clase molecular A; son las que presentan mayor diversidad y distribución. Estas $\beta$-lactamasas se caracterizan por hidrolizar carbapenémicos, penicilinas, cefalosporinas y aztreonam. Su actividad in vitro es pobremente inhibida por ácido clavulánico y tazobactam, y presenta una elevada inhibición por ácido fenilborónico y avibactam ${ }^{11,27}$. Los genes que codifican para carbapenemasas de clase A de importancia clínica en $P$. aeruginosa, son transferidos en plásmidos ${ }^{27}$.

La primera familia incluye las $\beta$-lactamasas tipo GES, de las que se han identificado dos variantes, GES2 y GES-5, entre aislados clínicos de $P$. aeruginosa y brotes nosocomiales en diferentes regiones geográficas; estas enzimas demuestran una extensión de su actividad hidrolítica que incluye a imipenem ${ }^{11}$.

La segunda familia incluye las carbapenemasas de $K$. pneumoniae (KPC); en la actualidad se han descrito 43 variantes alélicas del gen $b l a_{\mathrm{KPC}}$, las que difieren entre sí por 1- 3 aminoácidos ${ }^{27}$. Estas variantes de KPC han sido clasificadas desde $b l a_{\mathrm{KPC}-2}$ a $b l a_{\mathrm{KPC}-46}$. La primera $P$. aeruginosa productora de KPC se informó entre los aislados clínicos en Colombia, y se caracterizó como KPC-2. Posteriormente, se identificó KPC-2 entre aislados de $P$. aeruginosa de Puerto Rico, Trinidad y Tobago, China y los Estados Unidos de América (E.U.A.), y KPC-5 fue caracterizada en un aislado clínico de $P$. aeruginosa de Puerto Rico ${ }^{(11)}$. Las cepas de $P$. aeruginosa que producen KPC pueden mostrar resistencia a todos los antimicrobianos $\beta$-lactámicos, excepto a ceftazidima/avibactam ${ }^{20}$.

Son las $\beta$-lactamasas que pertenecen a la clase molecular D, llamadas también oxacilinasas que, adicionalmente a la hidrólisis de penicilinas, cefalosporinas y carbapenémicos, tienen la capacidad de hidrolizar oxacilina y cloxacilina. Hasta la fecha sólo se han descrito unas pocas carbapenemasas de tipo OXA en $P$. aeruginosa (Tabla 2). La carbapenemasa OXA-40 fue detectada en dos aislados clínicos no relacionados clonalmente, resistentes a imipenem, en España ${ }^{15}$; demostró ser idéntica a la enzima encontrada en Acinetobacter baumannii. Aunque bla ${ }_{O X A-40}$ fue descrita originalmente como un gen cromosómico en $A$. baumannii, puede encontrarse también en plásmidos conjugativos. Pseudomonas aeruginosa

\section{Grupo 3: Metalo- $\beta$-lactamasas (MBLs)}

Son enzimas de clase molecular B, se caracterizan por su requerimiento de uno o dos iones de zinc en su sitio activo; están mediados por plásmidos y son altamente transferibles ${ }^{13}$. Las MBLs se subdividen, a su vez, en tres subclases (B1, B2, B3), basado en identificación de secuencias de aminoácidos. Las enzimas de la subclase B1 y B3 tienen dos iones de zinc en su sitio activo y pueden hidrolizar penicilinas, cefalosporinas y carbapenémicos, en comparación con las enzimas de subclase B2 que tienen un ión de zinc. Estas enzimas hidrolizan 
solamente carbapenémicos y no hidrolizan penicilinas ni cefalosporinas ${ }^{16}$; tampoco hidrolizan eficientemente al aztreonam. Sin embargo, la co-producción de cefalosporinasas y/o carbapenemasas de clase A o D pueden conferir resistencia al aztreonam y su acción es inhibida por el agente quelante EDTA (ácido etilen-diaminotetra-acético $)^{11,27,28}$.

La primera MBL transferible, IMP-1, se identificó en 1988 en un aislado clínico de $P$. aeruginosa de Japón ${ }^{13}$. Desde este primer informe, se han descrito docenas de MBLs transferibles a nivel mundial, y pertenecen a cinco familias distintas: IMP, VIM, GIM, SPM y NDM-1. La mayoría de ellas han sido reportadas en Europa y Asia, encontrándose también algunas subclases de VIM en E.U.A.; en Latinoamérica se identificó y reportó por primera vez el gen bla ${ }_{V I M-2}$ que codifica para la MBL VIM-2, en una cepa de Pseudomonas fluorescens en Chile y 3 cepas de $P$. aeruginosa en Venezuela, resistentes a imipenem ${ }^{11,13,16,28,29}$.

\section{Detección de carbapenemasas en Pseudomonas aeruginosa}

La detección de la resistencia a carbapénemicos mediada por carbapenemasas tiene importancia clínica particular, porque permite optimizar la aplicación de medidas de control de infecciones (barreras de contacto) para contener su diseminación y ajustar el tratamiento teniendo en cuenta las nuevas alternativas terapéuticas. Algunas de las pruebas más empleadas se resumen en la Tabla 3.

\section{Resistencia enzimática a los aminoglucósidos}

\section{Enzimas modificadoras de aminoglucósidos (EMA)}

Los aminoglucósidos (gentamicina, amikacina, tobramicina, etc.), son un grupo de antimicrobianos comúnmente utilizados en el tratamiento de infecciones por $P$. aeruginosa, contienen un anillo aminociclitol unido a los aminoazúcares por enlaces glucosídicos y su actividad depende de la unión a un sitio altamente conservado del ARNr 16S. La resistencia a los aminoglucósidos se debe a múltiples factores, como la reducción de la permeabilidad de membrana, bombas de expulsión, mutaciones ribosomales y mecanismos enzimáticos (EMA) que modifican la estructura molecular de los grupos amino y glucósido $(\text { Tabla } 4)^{9,12,13}$. Las EMA inactivan los aminoglucósidos mediante la unión de grupos acetilo, fosfato o adenilo a los sustituyentes amino e hidroxilo en la molécula del antimicrobiano (Tabla 4). Estas modificaciones reducen significativamente la afinidad de los aminoglucósidos por la diana (subunidad ribosomal 30S) y bloquean su actividad $^{21}$.

Un gen común de la acetiltransferasa de resistencia a gentamicina de $P$. aeruginosa, aac (3) está asociado con un transposón localizado cromosómicamente (Tn801) que también porta un gen de $\beta$-lactamasa $\left(b_{\text {TEM-21 }}\right)$. De manera similar, el gen aph (3') que confiere resistencia a kanamicina, neomicina y estreptomicina, y el gen ant (3")-I que confiere resistencia a tobramicina, netilmicina y amikacina, se transportaron en elementos genéticos móviles (EGM) que se asocian con la resistencia a las $\beta$-lactamasas. Se ha informado que la prevalencia de

\begin{tabular}{|c|c|c|c|c|}
\hline Prueba & Desempeño & $\begin{array}{l}\text { Tiempo de } \\
\text { respuesta }\end{array}$ & Alcance & Limitaciones \\
\hline Sinergia con EDTA & $\begin{array}{l}\text { Sensibilidad: } 92 \% \\
\text { Especificidad: } 94 \%\end{array}$ & $16-20 \mathrm{~h}$ & $\begin{array}{l}\text { Diferenciación de metalo/ } \\
\text { carbapenemasas (clase B) }\end{array}$ & $\begin{array}{l}\text { Falsos positivos por permeabilización de la membrana. No } \\
\text { detecta co-producciones de enzimas (Clases } A+B \circ B+D \text { ). }\end{array}$ \\
\hline $\begin{array}{l}\text { Inmunoensayo de flujo } \\
\text { lateral }\end{array}$ & $\begin{array}{l}\text { Sensibilidad: } 100 \% \\
\text { Especificidad: } 95-100 \%\end{array}$ & $15 \mathrm{~min}$ & $\begin{array}{c}\text { Detección de carbapenemasas } \\
\text { de clases A (KPC), Clase B } \\
\text { (NDM/VIM/IMP) y D (OXA-48) }\end{array}$ & $\begin{array}{l}\text { Limitado a las variantes contenidas en el inserto del producto. } \\
\text { Mutaciones o nuevas variantes pueden no ser detectadas }\end{array}$ \\
\hline RPC Multiplex & $\begin{array}{l}\text { Sensibilidad: } 100 \% \\
\text { Especificidad: } 98,1 \%\end{array}$ & 1 hora & $\begin{array}{l}\text { Detección de carbapenemasas } \\
\text { de clases A (KPC), Clase B } \\
\text { (NDM/VIM/IMP) y D (OXA-48) }\end{array}$ & $\begin{array}{l}\text { Limitado a las variantes contenidas en el inserto del producto. } \\
\text { Mutaciones o nuevas variantes pueden no ser detectadas }\end{array}$ \\
\hline
\end{tabular}

Fuente: Villegas MV, y cols. 2016(26). Wozniak A, y cols. 201930. Abreviaciones: mCIM: modified carbapenem inactivation method, EDTA: ácido etilen-diamino-tetra-acético, KPC: Klebsiella pneumoniae carbapenemase, NDM: Nueva Delhi metalo carbapenemase, IMP: imipenemase, OXA: oxacilinase, RPC: reacción de polimerasa en cadena. 


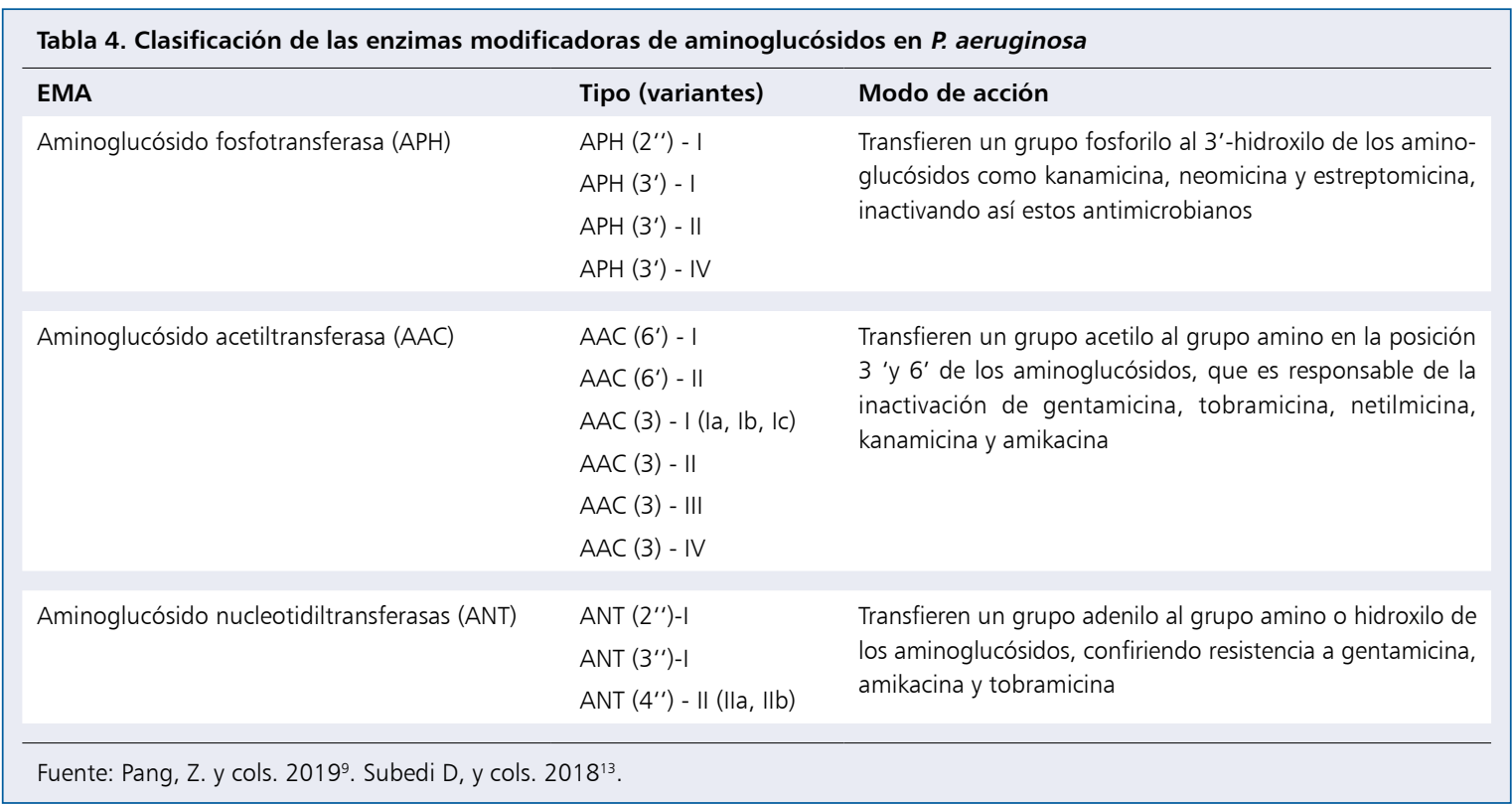

EMA en $P$. aeruginosa resistente a los aminoglucósidos es de hasta $75 \%{ }^{13}$.

\section{Metilasas del ARNr16S}

Este mecanismo de resistencia a aminoglucósidos se basa en la modificación del sitio diana. Los genes codificantes se mueven en elementos genéticos móviles (transposones, plásmidos conjugativos) lo que favorece su propagación horizontal. Los aislados que producen metilasas del ARNr16S son multi-resistentes por definición. Estos plásmidos conjugativos transportan genes codificantes de enzimas BLEEs y MBLs. Se han identificado diez metilasas de ARNr16S entre los aislados gramnegativos: ArmA, RmtA, RmtB, RmtC, RmtD, RmtD2, RmtE, RmtF, RmtG y NpmA ${ }^{12}$.

La primera metilasa ARNr16S recuperada en $P$. aeruginosa fue identificada en el año 2003, en un aislado clínico de Japón que producía RmtA. En el 2009 se identificaron en Corea del Sur, otras enzimas RmtA en aislados de $P$. aeruginosa. El gen $r m t A$ se encuentra en elementos genéticos móviles, como el transposón Tn5041. En el 2007, la metilasa RmtD se informó por primera vez en Brasil, a partir de un aislado clínico de P. aeruginosa panresistente que co-producía la MBL SPM-1. El gen r $m t D$ comparte $40 \%$ de identidad de aminoácidos con el gen $\mathrm{r} m t A$, y las estructuras genéticas que rodean a ambos genes correspondientes compartían características similares. Posteriormente, otro estudio subrayó que la co-producción de MBL SPM-1 y la metilasa de ARNr16S-RmtD era común entre los aislados de $P$. aeruginosa resistentes a imipenem recuperados en hospitales de São Paulo, Brasil ${ }^{12}$.

\section{Nuevas alternativas terapéuticas para el manejo de Pseudomonas aeruginosa MDR/ XDR}

Las nuevas opciones de tratamiento de $P$. aeruginosa MDR se basan en combinaciones de antimicrobianos antiguos y nuevos inhibidores, o en el desarrollo de nuevas moléculas sintéticas. Se busca con estos antimicrobianos, optimizar los desenlaces clínicos, minimizando los efectos secundarios y la actividad sub-óptima de las polimixinas $(\text { Tabla } 5)^{3}$.

\section{Cefalosporinas sideróforas (también conocidas como cefemas de catecol)}

\section{Cefiderocol}

Es una nueva cefalosporina siderófora con un residuo de catecol en la posición 3 de la cadena lateral, que permite la unión del ion férrico y el complejo resultante (iones cefiderocol y de hierro) penetra activamente las bacterias utilizando los sistemas transportadores de hierro y el influjo acelerado aumenta la actividad de cefiderocol contra bacterias gramnegativas, incluyendo cepas MDR productoras de $\beta$-lactamasas ${ }^{31}$. Se evaluó la actividad antibacteriana de cefiderocol contra bacterias gramnegativas no fermentadoras de glucosa como $A$. baumannii complex, $P$. aeruginosa y Stenotrophomonas maltophilia. Los resultados obtenidos indican que cefiderocol tiene actividad in vitro más potente contra bacterias no fermentadoras, con valores de CIM significativamente 


\begin{tabular}{|c|c|c|}
\hline Agentes & Fase de desarrollo & Indicaciones de uso \\
\hline \multicolumn{3}{|l|}{ Cefalosporinas sideróforas } \\
\hline cefiderocol & Aprobado por FDAJEMA para uso clínico & ITUC \\
\hline \multicolumn{3}{|c|}{ Cefalosporinas + inhibidor de $\beta$-lactamasa } \\
\hline ceftazidima/avibactam & Aprobado por FDAJEMA para uso clínico & ITUc, NN, NAV e IIAc + metronidazol \\
\hline ceftolozano/tazobactam & Aprobado por FDAJEMA para uso clínico & ITUc, NN, NAV y IIAc + metronidazol \\
\hline cefepima/tazobactam & Fase 3 & ITU c \\
\hline cefepima/zidebactam & Fase 1 & ND \\
\hline \multicolumn{3}{|c|}{ Carbapenem + inhibidor de $\beta$-lactamasa } \\
\hline imipenem/relebactam & Aprobado por FDA para uso clínico & ITUc y IIAc - \\
\hline \multicolumn{3}{|c|}{ Monobactam + inhibidor de $\beta$-lactamasa } \\
\hline aztreonam/avibactam & Fase 3 & ITUC, NN, NAV y IIAc + metronidazol \\
\hline \multicolumn{3}{|l|}{ Neoglucósidos } \\
\hline plazomicina & Aprobado por la FDA para el uso clínico & ITUc \\
\hline
\end{tabular}

más bajos en comparación con ceftazidima, meropenem, levofloxacina, cefepima y piperacilina/tazobactam. Cefiderocol mostró valores bajos de CIM para la mayoría de las cepas probadas, incluidas las cepas resistentes a los $\beta$-lactámicos, como las cepas que poseen MBL (GIM-1, IMP, SPM-1 y VIM) ${ }^{32}$. Para el tamizaje del antibiograma, existen puntos de corte CLSI de investigación y debe usarse Mueller Hinton con baja concentración de hierro ${ }^{10}$.

\section{Nuevos aminoglucósidos}

\section{Plazomicina}

Es un aminoglucósido semisintético de nueva generación, derivado del producto natural sisomicina. Los aminoglucósidos ejercen actividad contra bacterias gramnegativas al inhibir la síntesis de proteínas en la subunidad 30S del ribosoma; sin embargo, la resistencia que pueden presentar algunas bacterias a este tipo de antimicrobianos es debido principalmente a la producción de las EMA. Plazomicina es capaz de evadir un amplio espectro de EMA, pero es lábil a las metiltransferasas ribosomales $\mathrm{ARNr} 16 \mathrm{~S}$ que usualmente son transferidas en plásmidos conjugativos, donde también se movilizan las carbapenemasas como NDM. Plazomicina demuestra una potente actividad in vitro contra patógenos bacterianos gramnegativos y grampositivos ${ }^{33}$.

\section{Antimicrobianos $\beta$-lactámicos existentes combinados con nuevos inhibidores de $\beta$-lactamasas}

\section{Ceftazidima/avibactam (CZA)}

Resulta de la combinación de una cefalosporina antipseudomonas: ceftazidima, con el inhibidor de $\beta$-lactamasas avibactam. Fue aprobado por la Administración de Medicamentos y Alimentos (FDA, sigla en inglés) en 2015 y por la Agencia Europea de Medicinas (EMA, sigla en inglés), para el tratamiento de adultos con infecciones complicadas del tracto urinario (ITUc), incluyendo pielonefritis aguda, e infecciones intra abdominales complicadas (IIAc) en combinación con metronidazol, y fue aprobada recientemente por la FDA para el tratamiento de neumonía adquirida en el hospital (NAH) y neumonía asociada a ventilador (NAVM) a la misma dosis $(2,5 \mathrm{gm} \mathrm{c} / 8 \mathrm{~h} \text { en } 2 \mathrm{~h} \text { de infusión })^{34,35}$. En Europa, además tiene la indicación para el manejo de infecciones por bacterias gramnegativas aerobias en pacientes adultos con limitadas opciones terapéuticas ${ }^{34}$.

Ceftazidima es una cefalosporina de tercera generación de amplio espectro que, al igual que otros $\beta$-lactámicos, ejerce su efecto al unirse a las proteínas de unión a penicilina (PBP), inhibiendo así el entrecruzamiento del peptidoglicano durante la síntesis de la pared celular, conduciendo a la lisis y muerte 
0,25 a $0,5 \mu \mathrm{g} / \mathrm{ml}$ con la adición de relebactam. Se observó una potenciación dependiente de la concentración de imipenem por relebactam para todos los grupos de $P$. aeruginosa resistentes a imipenem, excepto aquellos con MBL. Para aislados que carecen de OprD, y sin otros mecanismos de resistencia, las CIM de imipenem fueron 16 a $64 \mu \mathrm{g} / \mathrm{ml}$ y con la adición de relebactam se redujeron $\mathrm{a} \leq 2 \mu \mathrm{g} / \mathrm{ml}$ en $4 / 8$ casos, y en $7 / 8$ casos a $8 \mu \mathrm{g} / \mathrm{ml}^{39}$.

Lapuebla y cols., encontraron en 490 aislados de $P$. aeruginosa, que la adición de relebactam a imipenem redujo la CIM de 2-16 a 0,5-2 $\mu \mathrm{g} / \mathrm{ml}$. Así mismo, encontraron que en 144 aislados no susceptibles a imipenem, con la adición de relebactam se obtuvieron valores de las CIM entre $1-2 \mu \mathrm{g} / \mathrm{ml}^{40}$.

observado en aislados que producen MBL de clase (VIM, NDM, IMP), sobre los cuales avibactam no tiene actividad inhibitoria o en cepas con mutaciones en el gen $A m p C$, mientras que la disminución de la permeabilidad y la expresión de bombas de expulsión, contribuyen a la resistencia a ceftazidima, independientemente de la presencia de avibactam ${ }^{(35,37)}$. Se evaluó recientemente la actividad en paralelo de ceftolozano/tazobactam $(\mathrm{C} / \mathrm{T})$ y ceftazidima/avibactam en 195 cepas de $P$. aeruginosa resistentes a meropenem procedentes de la vía respiratoria, en las que, las actividades inhibitorias de CZA y $\mathrm{C} / \mathrm{T}$ fueron de 81 y $91 \%$, respectivamente ${ }^{37}$.

\section{Imipenem/relebactam}

Relebactam es un inhibidor de $\beta$-lactamasas diazobiciclo-octano no $\beta$-lactámico, está relacionado estructuralmente con avibactam, diferenciándose de éste por presentar un anillo adicional de piperidina. La piperidina posee carga positiva, permitiéndole resistir a las bombas de expulsión de las bacterias. Relebactam es efectivo contra $\beta$-lactamasas de clase A (ej. KPC) y clase C (ej. AmpC), pero es inactivo frente a $\beta$-lactamasas de clase B (ej. VIM, NDM e IMP) y $\beta$-lactamasas clase D (ej. OXA-48). La adición de relebactam mejora la actividad de imipenem e inhibe las enzimas carbapenemasas por acetilación, junto con la inhibición de la síntesis de la pared celular y es altamente activo frente a $\beta$-lactamasa PER-2, PDC-3 y BLEE de $P$. aeruginos $a^{34,38}$. Estudios ha demostrado que relebactam tiene una actividad más potente contra PER-2, una $\beta$-lactamasa producida por $P$. aeruginosa, en comparación con avibactam $\left(\mathrm{K}_{\mathrm{i} \text { app }} 5,8\right.$ $\pm 0,6$ y $29 \pm 3 \mu \mathrm{M}$, respectivamente). Sin embargo, al comparar imipenem/relebactam con ceftazidima/ avibactam, ceftazidima es hidrolizada por PER a diferencia de imipenem. En un estudio similar, se determinó que tanto relebactam como avibactam tienen actividad contra PDC-3, una BLEE producida por $P$. aeruginosa $\left(\mathrm{K}_{\mathrm{i} \text { app }}\right.$ $3,4 \pm 0,4$ y $2,5 \pm 0,3 \mu \mathrm{M}$, respectivamente $)^{38}$.

En estudios realizados, se demostró que las CIM de imipenem para cepas de $P$. aeruginosa susceptibles fueron principalmente de 1 a $2 \mu \mathrm{g} / \mathrm{ml}$ y se redujeron a

\section{Nuevos antimicrobianos $\beta$-lactámicos combinados con inhibidores de $\beta$-lactamasa existentes}

\section{Ceftolozano/tazobactam (C/T)}

Es la combinación de una nueva cefalosporina antipseudomonas de espectro extendido, con un conocido inhibidor de $\beta$-lactamasas. Fue aprobado por la FDA en diciembre de 2014 para el tratamiento de infecciones intra-abdominales complicadas más metronidazol, e infecciones complicadas del tracto urinario, incluyendo pielonefritis aguda, en pacientes adultos ${ }^{3,34}$. En 2019 recibió la aprobación FDA para neumonía nosocomial incluyendo NAVM. Al igual que con todos los $\beta$-lactámicos, la actividad antimicrobiana se debe a la inhibición de las PBP involucradas en los pasos finales de la biosíntesis de peptidoglicanos ${ }^{35}$.

La molécula de ceftolozano es una oximino-cefalosporina que difiere de ceftazidima principalmente por la presencia de una cadena lateral más voluminosa en la posición 3 del anillo de dihidrotiazina. Esta modificación conlleva una afinidad más alta y un perfil de inhibición más amplio hacia las PBP esenciales de $P$. aeruginosa (PBP1b, PBP1c, PBP2 y PBP3) en comparación con ceftazidima, mientras que la afinidad por PBP4 sigue siendo inferior a la de imipenem y, por lo tanto, no inducirá una sobre-expresión de AmpC. Debido a esta modificación, ceftolozano también es más estable frente a la $\beta$-lactamasa AmpC cromosómica de $P$. aeruginosa y es un sustrato, en general pobre, de las bombas de expulsión MexAB-OprM encontradas en esta especie ${ }^{36}$.

Gracias a estas características y al hecho de que, a diferencia de los carbapenémicos, la entrada a través de la membrana externa de $P$. aeruginosa no se ve afectada por la funcionalidad de la porina OprD, ceftolozano exhibe una actividad antipseudomonas superior. El tazobactam le confiere protección frente a algunas $\beta$-lactamasas tipo serina $^{19,32}$. 
La actividad in vitro de ceftolozano es ocho veces más alta que la de ceftazidima y se ha demostrado que $\mathrm{C} / \mathrm{T}$ tiene actividad in vitro superior a imipenem y piperacilina/ tazobactam. Un aspecto importante que se ha determinado en estudios in vitro, es que contra $P$. aeruginosa resistente a ceftazidima, $\mathrm{C} / \mathrm{T}$ mantiene su actividad, resaltando la utilidad clínica de $\mathrm{C} / \mathrm{T}$ contra las infecciones por este microorganismo. Además, los estudios han demostrado que las $\mathrm{CIM}$ de $\mathrm{C} / \mathrm{T}$ frente a aislados de P. aeruginosa que producen $\beta$-lactamasas AmpC se encontraban entre 2 y $4 \mu \mathrm{g} / \mathrm{ml}$, lo que sugiere estabilidad de $\mathrm{C} / \mathrm{T}$ frente a estas enzimas ${ }^{34}$. Ceftolozano ha demostrado una potente actividad in vitro contra $P$. aeruginosa $\left(\mathrm{CIM}_{50 / 90}, 0,5 / 4\right.$ $\mu \mathrm{g} / \mathrm{ml}$ ) y se ha demostrado que $\mathrm{C} / \mathrm{T}$ tiene mayor actividad en comparación con piperacilina/tazobactam, ceftazidima o meropenem. Así mismo, se ha demostrado que $\mathrm{C} / \mathrm{T}$ permanece activo contra la mayoría de las cepas de MRD / XDR ( $\left.\mathrm{CIMc}_{50 / 90}, 4 />64 \mu \mathrm{g} / \mathrm{ml}\right)$, ya que no se ve afectado por algunos de los principales mecanismos de resistencia en $P$. aeruginosa (hiperproducción de AmpC, bombas de eflujo y/o pérdida de OprD).

Los estudios in vitro han demostrado que el desarrollo de resistencia a ceftolozano / tazobactam es mucho más lento que el de resistencia a otros agentes antipseudomonas (ej. ceftazidima).

En Latinoamérica el principal mecanismo de resistencia a ceftolozano es la presencia de carbapenemasas de clase A (principalmente KPC) y de clase B ( principalmente VIM $)^{(34)}$.

\section{Antibiograma en Pseudomonas aeruginosa}

Para el uso apropiado de antimicrobianos antipseudomonas se requiere conocer la epidemiología local, los mecanismos de resistencia circulantes, las indicaciones, alcances y limitaciones de todas las moléculas disponibles, incluyendo las nuevas alternativas y conocer el impacto sobre la presión selectiva de los antimicrobianos antipseudomonas para elegir la terapia con menor daño colateral. Adicionalmente, realizar las pruebas confirmatorias para mecanismos de resistencia enzimática a $\beta$-lactámicos con relevancia clínica (carbapenemasas) ${ }^{26}$. En la Tabla 6, se presentan cinco recomendaciones para una interpretación adecuada del antibiograma en el contexto de resistencia enzimática y no enzimática a los $\beta$-lactámicos en el marco de un PROA.

\section{Conclusiones}

El continuo aumento de pacientes inmunocomprometidos y la ventaja evolutiva de las bacterias para mutar rápidamente y adaptarse a las amenazas antimicrobianas/biocidas en su entorno, hacen que el tratamiento de las enfermedades infecciosas sea un desafío serio. Este es el caso de $P$. aeruginosa, patógeno oportunista con gran capacidad para desarrollar multi-resistencia. Si bien la diseminación de mecanismos por elementos genéticos móviles es un constante desafío para el control de infecciones, quizás el reto más difícil que

\section{Tabla 6. Reglas de experto en la interpretación de antibiograma para $P$. aeruginosa}

\section{Regla Comentarios}

1. Suprimir antimicrobianos con resistencia intrínseca

2. Tamizar todos los antimicrobianos antipseudomonas

3. Incluir la CIM en el informe del antibiograma

4. Detectar mecanismos de resistencia con relevancia clínica

5. Editar los informes e incluir pies de página
Suprimir antimicrobianos con resistencia natural en $P$. aeruginosa permitirá que se escojan únicamente las moléculas activas sobre este microorganismo. La lista completa de antimicrobianos con resistencia natural se encuentra en la Tabla 1.

Los espectros hidrolíticos de los diferentes tipos de enzima pueden variar de acuerdo con su nivel de expresión y la adquisición de elementos genéticos móviles. Adicionalmente, es importante tener alternativas disponibles en casos de multi-resistencia. Los antimicrobianos antipseudomonas más usados son:ceftazidima, cefepima, aztreonam, piperacilina/tazobactam, imipenem, meropenem, doripenem, ceftolozano/tazobactam, ceftazidima/avibactam, ciprofloxacina, levofloxacina, gentamicina, amikacina, colistina, fosfomicina

La CIM es fundamental para escoger la alternativa con mayor potencia y apoya la detección de mecanismos de resistencia con relevancia clínica. La CIM debe incluirse al menos para $\beta$-lactámicos, quinolonas y polimixinas

La detección y diferenciación de carbapenemasas permite escoger las alternativas terapéuticas más adecuadas como ceftazidima/ avibactam, aztreonam, combinaciones de antimicrobianos, etc, o permite el uso de otras moléculas como ceftolozano/tazobactam para resistencia no mediada por carbapenemasas

Las ediciones permiten una interpretación correcta del antibiograma. Por ejemplo, en caso de resistencia a ciprofloxacina, cambiar a resistente la interpretación de otras quinolonas como levofloxacina. Incluir notas informando la presencia de las carbapenemasas y editar los informes cuando se tengan resultados moleculares o fenotípicos rápidos. Por ejemplo: informar resistencia a piperacilina/ tazobactam, cefepima o aztreonam cuando se detecten carbapenemasas de clase A (KPC) 
enfrentamos con este patógeno es su rápida capacidad de desarrollar resistencia a varias clases de antimicrobianos simultáneamente, inclusive durante el tratamiento de cepas inicialmente sensibles e incluyendo a las nuevas moléculas.

El laboratorio de microbiología juega un papel importante en la búsqueda de pruebas más sensibles, específicas y oportunas, para detectar estos mecanismos de resistencia, por lo que la estrecha colaboración entre el laboratorio y el médico clínico es esencial para el cuidado del paciente, y es la base del PROA.
Al ser la resistencia enzimática uno de los principales mecanismos de resistencia expresados por $P$. aeruginosa, se necesita el desarrollo de nuevos agentes antimicrobianos que sean capaces de bloquear o inhibir estas enzimas. De los nuevos antimicrobianos que han sido aprobados y otros que están en desarrollo, se puede concluir que ninguno es activo frente a cepas productoras de MBL, que son las enzimas más frecuentemente encontradas en esta especie. Por lo tanto, combinaciones de inhibidores como avibactam + aztreonam pueden ser una alternativa para el manejo de estas infecciones.

\section{Referencias bibliográficas}

1.- Morita Y, Tomida J, Kawamura Y. Responses of Pseudomonas aeruginosa to antimicrobials Front Microbiol. 2014; 4 (Jan): 1-8. doi: $10.3389 /$ fmicb.2013.00422.

2.- Oliver A. Epidemiology and carbapenem resistance mechanisms in Pseudomonas aeruginosa: Role of high-risk clones in multidrug resistance. Enf Infec Microbiol Clin. 2017; 35 (3): 137-8. doi: 10.1016/j. eimc.2016.11.006.

3.- Ruiz-Garbajosa P, Cantón R. Epidemiology of antibiotic resistance in Pseudomonas aeruginosa. Implications for empiric and definitive therapy. Rev Esp Quimioter. 2017; 30 (Suppl 1): 8-12. PMID: 28882007.

4.- Villa L M, Cortés J A, Leal A L, Meneses A, Meléndez M P. Pseudomonas aeruginosa resistente a antimicrobianos en hospitales colombianos. Rev Chil Infectol. 2013; 30 (6): 605-10. http://dx.doi.org/10.4067/S071610182013000600005.

5.- Yadav R, Bulitta J B, Wang J, Nation R L, Landersdorfer C B. Evaluation of pharmacokinetic/pharmacodynamic modelbased optimized combination regimens against multidrug-resistant Pseudomonas aeruginosa in a murine thigh infection model by using humanized dosing schemes. Antimicrob Agents Chemother. 2017; 61 (12): 1-11. doi: 10.1128/ AAC.01268-17.

6.- Bolaños C, Iannacone J. Patrones fenotípicos de resistencia en Pseudomonas aeruginosa de muestras clínicas a nivel de Sudamérica. ReserachGate. 2016; 1 (2): 1-29. doi: 10.24039/ cv20164164.

7.- Estepa V, Rojo-Bezares B, Azcona-Gutiérrez J M, Olarte I, Torres C, Sáenz Y. Caracterización de mecanismos de resistencia a carbapenémicos en aislados clínicos de Pseudomonas aeruginosa en un hospital español. Enferm Infecc Microbiol Clin. 2017; 35 (3): 141-7. doi: 10.1016/j.eimc.2015.12.014.

8.- De Matos E C O, Andriolo R B, Rodrigues Y C, Lima P D L de, Carneiro I C do R S, Lima K V
B. Mortality in patients with multidrug-resistant Pseudomonas aeruginosa infections: a metaanalysis. Rev Soc Bras Med Trop. 2018; 51 (4): 415-20. doi: 10.1590/0037-8682-0506-2017.

9.- Pang Z, Raudonis R, Glick B R, Lin $\mathrm{T} J$, Cheng Z. Antibiotic resistance in Pseudomonas aeruginosa: mechanisms and alternative therapeutic strategies. Biotechnol Adv. 2019; 37 (1): 177-92. doi: 10.1016/j. biotechadv.2018.11.013.

10.- CLSI. Performance Standards for Antimicrobial Susceptibility Testing. 30th ed. CLSI supplement M100,, editor. Wayne, PA: Clinical and Laboratory Standards Institute. 2020; 1-332. https://clsi.org/standards/products/ microbiology/documents/m100/

11.- Wolter D, Lister P. Mechanisms of $\beta$-lactam resistance among Pseudomonas aeruginosa. Curr Pharm Des. 2012; 19 (2): 209-22. PMID: 22894618.

12.- Potron A, Poirel L, Nordmann P. Emerging broad-spectrum resistance in Pseudomonas aeruginosa and Acinetobacter baumannii: Mechanisms and epidemiology. ELSEVIER. 2015; 45 (6): 568-85. doi: 10.1016/j. ijantimicag.2015.03.001.

13.- Subedi D, Vijay A K, Willcox M. Overview of mechanisms of antibiotic resistance in Pseudomonas aeruginosa: an ocular perspective. Clin Exp Optom. 2018; 101 (2): 162-71. doi: 10.1111/cxo.12621.

14.- Shaikh S, Fatima J, Shakil S, Rizvi S M D, Kamal M A. Antibiotic resistance and extended spectrum beta-lactamases: types, epidemiology and treatment. Saudi J Biol Sci. 2015; 22 (1): 90-101. doi: 10.1016/j.sjbs.2014.08.002.

15.- Bonnin R, Bogaerts P, Girlich D, Huang T D, Laurent D, Glupczynski Y, et al. Molecular characterization of OXA-198 carbapenemaseproducing Pseudomonas aeruginosa clinical isolates. Am Soc Microbiol. 2018; 62 (6): 1-7. doi: 10.1128/AAC.02496-17.

16.- Wu W, Feng Y, Tang G, Qiao F, McNally A, Zong $Z$. NDM metallo- $\beta$-lactamases and their bacterial producers in health care settings. Clin Microbiol Rev. 2019; 32 (2): 1-45. doi: 10.1128/CMR.00115-18

17.- Laudy A E, Róg P, Smolinska-Król K, Ćmiel M, Søoczynska A, Patzer J, et al. Prevalence of ESBL-producing Pseudomonas aeruginosa isolates in Warsaw, Poland, detected by various phenotypic and genotypic methods. PLoS One. 2017; 12 (6): 1-15. https://doi.org/10.1371/ journal.pone.0180121.

18.- Lister P, Wolter D, Hanson N. Antibacterialresistant Pseudomonas aeruginosa: clinical impact and complex regulation of chromosomally encoded resistance mechanisms. Am Soc Microbiol. 2009; 22 (4): 582-610. doi: 10.1128/CMR.00040-09.

19.- Horcajada J P, Montero M, Oliver A, Sorlí L, Luque S, Gómez-Zorrilla S, et al. Epidemiology and treatment of multidrug-resistant and extensively drug-resistant Pseudomonas aeruginosa infections. Clin Microbiol Rev. 2019; 32 (4): 1-52. doi: 10.1128/CMR.0003119.

20.- Humphries R, Hindler J, Wong-Beringer A, Miller S. Activity of ceftolozane-tazobactam and ceftazidime-avibactam against beta-lactamresistant Pseudomonas aeruginosa isolates. Antimicrob Agents Chemother 2017; 61 (12): e01858-17. doi: 10.1128/AAC.01858-17.

21.- Ullah W, Qasim M, Rahman H, Bari F, Khan S, Dworeck T, et al. Molecular identification of TEM-116 beta-lactamase gene in isolates of pathogenic Pseudomonas aeruginosa: A first report from Pakistan. Trop J Pharm Res. 2017; 16 (1): 149-54. http://dx.doi.org/10.4314/tjpr. v16i1.19.

22.- Bassetti M, Vena A, Croxatto A, Righi E, Guery B. How to manage Pseudomonas aeruginosa infections. Drugs Context. 2018; 7: 212527. doi: $10.7573 /$ dic. 212527 .

23.- Ullah W, Qasim M, Rahman H, Khan S, Rehman Z ur, Ali N, et al. CTX-M-15 and OXA-10 beta lactamases in multi drug resistant Pseudomonas aeruginosa: First report from Pakistan. Microb Pathog. 2017; 105: 240-4. doi: 10.1016/j.micpath.2017.02.039.

24.- Sales A, Fathi R, Mobaiyen H, Bonab F, Kondlaji K, Sadeghnezhadi M. Molecular study 
of efflux genes in Pseudomonas aeruginosa isolated from clinical samples. Int J Curr Microbiol Appl Sci. 2017; 6 (7): 4549-56. doI: 10.20546/ijcmas.2017.607.475.

25.- Croughs P D, Klaassen C H W, van Rosmalen J, Maghdid D M, Boers S A, Hays J P, et al. Unexpected mechanisms of resistance in Dutch Pseudomonas aeruginosa isolates collected during 14 years of surveillance. Int J Antimicrob Agents [Internet]. 2018; 52 (3): 407-10. DOI: 10.1016/j. ijantimicag.2018.05.009.

26.- Villegas M V, Zurita J, Esparza G. Guía para la implementación de un programa de optimización de antimicrobianos (PROA) a nivel hospitalario. Asociación Panamericana de Infectología 2016; 1-91. https://www.apiinfectologia.org/guia-para-laimplementacion-del-proa-a-nivel-hospitalario/

27.- Vera-Leiva A, Barría-Loaiza C, CarrascoAnabalón S, Lima C, Aguayo-Reyes A, Domínguez M, et al. KPC: Klebsiella pneumoniae carbapenemasa, principal carbapenemasa en enterobacterias. Rev Chil infectología 2012; 34 (5): 476-84. http://dx.doi. org/10.4067/S0716-10182017000500476.

28.- Pérez I A, García C P, Poggi M H, Braun J S, Castillo V C, Román JC, et al. Presence of metallo $\beta$-lactamases in imipenem-resistant Pseudomonas aeruginosa. Rev Med Chile 2008; 136 (4): 423-32. PMID: 18769784.

29.- Labarca J A, Salles M J C, Seas C, GuzmánBlanco M. Carbapenem resistance in Pseudomonas aeruginosa and Acinetobacter baumannii in the nosocomial setting in Latin America. Crit Rev Microbiol. 2016; 42 (2): 276-92. doi: 10.3109/1040841X.2014.940494.

30.- Wozniak A, Paillavil B, Legarraga P, Zumarán C, Prado S, García P. Evaluation of a rapid immunochromatographic test for detection of KPC in clinical isolates of Enterobacteriaceae and Pseudomonas species. Diagn Microbiol Infect Dis. 2019; 95 (2): 131-3. doi: 10.1016/j. diagmicrobio.2019.05.009. Epub 2019 May 24.

31.- Wright H, Bonomo R A, Paterson D L. New agents for the treatment of infections with Gram-negative bacteria: restoring the miracle or false dawn? Clin Microbiol Infect. 2017; 23 (10): 704-12. doi: 10.1016/j.cmi.2017.09.001.

32.- Ito A, Kohira N, Bouchillon S K, West J, Rittenhouse S, Sader H S, et al. In vitro antimicrobial activity of S-649266, a catecholsubstituted siderophore cephalosporin, when tested against non-fermenting Gram-negative bacteria. J Antimicrob Chemother. 2016; 71 (3): 670-7. doi: $10.1093 / \mathrm{jac} / \mathrm{dkv} 402$.

33.- Doi Y. Treatment options for carbapenemresistant gram-negative bacterial infections. Clin Infect Dis. 2019; 69 (Suppl 7): S565-75. doi: 10.1093/cid/ciz830.

34.- Pragasam A, Veeraraghavan B, Nalini E, Anandan S, Kaye K. An update on antimicrobial resistance and the role of newer antimicrobial agents for Pseudomonas aeruginosa. Indian J Med Microbiol. 2018; 36 (3): 303. doi: 10.4103/ijmm.IJMM_18_334.

35.- Shirley M. Ceftazidime-Avibactam: a review in the treatment of serious gram-negative bacterial infections. Indian J Med Microbiol. 2018; 78 (6): 675-92. doi: 10.1007/s40265-018-0902-x.

36.- Humphries R M, Hindler J A, Wong-Beringer A, Miller S A. Activity of ceftolozanetazobactam and ceftazidime-avibactam against beta-lactam-resistant Pseudomonas aeruginosa isolates. Antimicrob Agents Chemother. 2017; 61 (12): 1-4. doi: 10.1128/AAC.01858-17.

37.- Grupper M, Sutherland C, Nicolau D P. Multicenter Evaluation of ceftazidimeavibactam and ceftolozane-tazobactam inhibitory activity against meropenemnonsusceptible Pseudomonas aeruginosa from blood, respiratory tract, and wounds. Antimirob Agnts Chemother. 2017; 61 (10): e00875-17. doi: 10.1128/AAC.00875-17.

38.- Zhanel G G, Lawrence C K, Adam H, Schweizer F, Zelenitsky S, Zhanel M, et al. Imipenem-relebactam and meropenemvaborbactam: two novel carbapenem- $\beta$ lactamase inhibitor combinations. Drugs. 2018; 78 (1): 65-98. doi: 10.1007/s40265-0170851-9.

39.- Livermore D M, Warner M, Mushtaq S. Activity of MK-7655 combined with imipenem against Enterobacteriaceae and Pseudomonas aeruginosa. J Antimicrob Chemother. 2013; 68 (10): 2286-990. doi: 10.1093/jac/dkt178.

40.- Lapuebla A, Abdallah M, Olafisoye O, Cortes C, Urban C, Landman D, et al. Activity of imipenem with relebactam against gramnegative pathogens from New York City. Am Soc Microbiol. 2015; 59 (8): 5029-31. doi: 10.1128/AAC.00830-15. 